\title{
Prospects for dark energy evolution: a frequentist multi-probe approach
}

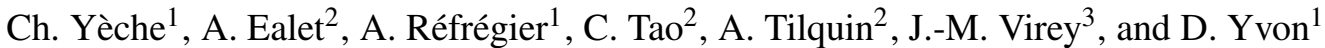 \\ ${ }^{1}$ DSM/DAPNIA, CEA/Saclay, 91191 Gif-sur-Yvette, France \\ e-mail: yeche@hep.saclay.cea.fr \\ 2 Centre de Physique des Particules de Marseille, CNRS/IN2P3-Luminy and Université de la Méditerranée, Case 907, \\ 13288 Marseille Cedex 9, France \\ 3 Centre de Physique Théorique ${ }^{\star}$, CNRS-Luminy and Université de Provence, Case 907, 13288 Marseille Cedex 9, France
}

Received 7 July 2005 / Accepted 24 October 2005

\begin{abstract}
A major quest in cosmology is to understand the nature of dark energy. It is now well known that the use of several cosmological probes is required to break the underlying degeneracies on cosmological parameters. In this paper, we present a method based on a frequentist approach that combines probes without any prior constraints. As one application, the current supernovae type Ia and cosmic microwave background data are analyzed with an evolving dark energy component, and our results are first compared to other analyses. We emphasize the consequences of implementing the dark energy perturbations for an equation of state that varies with time. We then simulate the expectations of different future projects. The constraints from weak lensing surveys on the measurement of dark energy evolution are combined with the measurements from the cosmic microwave background and type Ia supernovae. We present the impacts for mid-term and long-term surveys and confirm that the combination with weak lensing is very powerful in breaking parameter degeneracies. A second generation of experiments is, however, required to achieve a 0.1 error on the parameters describing the evolution of dark energy.
\end{abstract}

Key words. cosmological parameters - cosmic microwave background - large-scale structure of universe - dark matter - equation of state

\section{Introduction}

Supernovae type Ia (SNIa) observations (Knop et al. 2003; Riess et al. 2004) provide strong evidence that the universe is accelerating, in very good agreement with the combined constraints from the WMAP cosmic microwave background (CMB) (Bennett et al. 2003; Spergel et al. 2003) and large scale structures (Hawkins et al. 2003; Tegmark et al. 2004) observations. The simplest way to explain the present acceleration is to introduce a cosmological constant into Einstein's equations. In conjunction with cold dark matter, it forms the so-called $\Lambda \mathrm{CDM}$ model. Even if this solution agrees with current data, the measured value of the cosmological constant is very low compared to the vacuum energy expectations from particle physics, requiring a difficult fine tuning. A favorite solution to this problem involves the introduction of a new component, called "dark energy", which can be a scalar field as in quintessence models (Wetterich 1988; Peebles \& Ratra 1988).

The most common way to study this component is to measure its "equation of state" (EOS) parameter, defined as

^ "Centre de Physique Théorique" is UMR 6207 - "Unité Mixte de Recherche" of CNRS and of the Universities "de Provence", "de la Méditerranée" and "du Sud Toulon-Var" - Laboratory affiliated with FRUMAM (FR 2291). $w=p / \rho$, where $p$ is the pressure and $\rho$ the energy density of the dark energy. Most models predict an evolving equation $w(z)$. It has been shown (e.g., Maor et al. 2001, 2002; Virey et al. 2004a; Gerke \& Efstathiou 2002) that neglecting such an evolution biases the discrimination between $\triangle \mathrm{CDM}$ and other models. Analysis of dark energy properties needs to take time evolution (or redshift $z$ dependence) into account.

Other attractive solutions to the cosmological constant problem imply a modification of gravity (for a review, cf., e.g., Lue et al. 2004; or Carroll et al. 2005, and references therein). In this case, there is no dark energy as such and thus no dark energy equation of state. In this paper, we consider only the dark energy solution, keeping in mind that Lue et al. (2004), among others, have shown that the induced changes in the Friedmann equations could be parameterized in ways that are very similar to a dark energy evolving solution.

As various authors have noted (e.g., Huterer \& Turner 2001; Weller \& Albrecht 2002), SNIa observations alone will not be able to distinguish between an evolving equation of state and $\Lambda$ CDM. This technique indeed requires prior knowledge of the values of some parameters. In particular, precision in the measurement of the prior matter density $\Omega_{\mathrm{m}}$ has an impact on the constraints on the time evolution of the equation of state $w$, 
even in the simplest flat Universe cosmology (e.g., Virey et al. 2004b).

Extracting dark energy properties thus requires a combined analysis of complementary data sets. This can be done by using SNIa data in conjunction with other probes such as the $\mathrm{CMB}$, the large scale distributions of galaxies, Lyman $\alpha$ forest data, and the observations from large scale structures with the Sunyaev-Zeldovich effect (SZ) (Sunyaev \& Zeldovich 1980). In the near future, weak gravitational lensing surveys (WL) can also be used and will provide a unique method to directly map the distribution of dark matter in the universe (for reviews, cf., e.g., Bartelmann \& Schneider 2001; Mellier et al. 2002; Hoekstra et al. 2002; Refregier 2003; Heymans et al. 2005, and references therein).

Many combinations have already been performed with different types of data and procedures (e.g., Bridle et al. 2003; Douspis et al. 2003; Melchiorri et al. 2003; Wang \& Tegmark 2004; Tegmark et al. 2004; Upadhye et al. 2004; Ishak 2005; Seljak et al. 2004; Corasaniti et al. 2004; Xia et al. 2004). All studies have shown the consistency of existing data sets with the $\Lambda \mathrm{CDM}$ model and the complementarity of the different data sets in breaking degeneracies and constraining dark energy for future experiments. But the results differ by as much as $2 \sigma$ for the central values of the parameters describing an evolving equation of state.

In this paper, we choose three probes, namely, SNIa, CMB, and weak lensing, which seem to best constrain the parameters of an evolving equation of state when their results are merged. Considering a flat Universe, we combine the data in a coherent way, that is to say, under identical assumptions for the dark energy properties for the three probes, and we completely avoid the use of priors. This has not always been done systematically in all previous works. We also adopt a frequentist approach for the data combination, where the full correlations between the cosmological parameters are taken into account without any prior constraints. This method allows us to provide, simultaneously, confidence intervals on a large number of distinct cosmological parameters. Moreover, this approach is very flexible as it is easy to add or remove parameters in contrast to other methods.

The paper is organized as follows: in Sect. 2, we describe our framework and statistical procedure based on a frequentist approach, which can accommodate all parameters without marginalization. For our simulation and analysis, we use the CMBEASY package for CMB (Doran 2003), the Kosmoshow program for SNIa (Tilquin 2003), and an extension of the calculations from Refregier et al. (2003) for weak lensing. In each case, the programs take into account the time evolution of the equation of state (cf. Sect. 2.2 for details).

In Sect. 3, we apply this method to current data sets of SNIa and WMAP data. We give our results, compare them with other works and discuss the differences. In particular, we discuss how the treatment of the dark energy perturbations can explain some of the differences found in the literature. We also verify that the constraints on the cosmological parameters estimated with a Fisher matrix technique (Fisher 1935) are consistent with those obtained with a complete error analysis.
In Sect. 4, we simulate the statistical sensitivities of different combinations of future surveys. We present the expectations for the ground surveys from the Canadian French Hawaii Telescope Legacy Surveys (CFHTLS) and new CMB data from Olimpo, as well as the longer term Planck and SNAP space missions. For these future experiments, the results are combined with a Fisher matrix technique, and then compared and discussed.

Finally, our conclusions are summarized in Sect. 5.

\section{Combination method}

In this section, we first summarize the framework used in this paper, and describe our approach based on frequentist statistics.

\subsection{Dark energy parameterization}

The evolution of the expansion parameter is given by the Hubble parameter $H$ through the Friedmann equation

$\left(\frac{H(z)}{H_{0}}\right)^{2}=(1+z)^{3} \Omega_{\mathrm{m}}+\frac{\rho_{\mathrm{X}}(z)}{\rho_{\mathrm{X}}(0)} \Omega_{\mathrm{X}}+(1+z)^{2} \Omega_{k}$,

with

$\frac{\rho_{\mathrm{X}}(z)}{\rho_{\mathrm{X}}(0)}=\exp \left[3 \int_{0}^{z}\left(1+w\left(z^{\prime}\right)\right) \mathrm{d} \ln \left(1+z^{\prime}\right)\right]$

where the ratio of the dark energy density to the critical density is denoted $\Omega_{\mathrm{X}}$ in a general model and $\Omega_{\Lambda}$ in the simplest case of a Cosmological Constant $(w=-1)$. Also, $\Omega_{\mathrm{M}}$ is the corresponding parameter for (baryonic+cold dark) matter. Note that we have neglected the radiation component $\Omega_{R}$. The present total and curvature density parameters are $\Omega$ and $\Omega_{\kappa}=1-\Omega$, respectively. The present value of the Hubble constant is parameterized as $H_{0}=100 \mathrm{hm} \mathrm{s}^{-1} \mathrm{Mpc}^{-1}$.

As it is not possible to constrain a completely unknown functional form $w(z)$ of the time evolution of the equation of state, we adopt a parametric representation of the $z$ dependence of the equation of state. We need this parametric form to fit all the data sets over a large range of $z$ : from $z \simeq 0-2$ for the SNIa and weak lensing, up to $z \simeq 1100$ for the CMB. For this purpose, we choose the parameterization proposed by Chevallier \& Polarski (2001) and Linder (2003):

$w(z)=w_{0}+w_{a} z /(1+z)$,

which has an adequate asymptotic behavior. In this paper, we thus use two parameters, $w_{0}$ and $w_{a}$, to describe the time evolution of the equation of state (see justifications in Linder \& Huterer 2005). For this parameterization of $w(z)$, Eq. (2) reduces to:

$\rho_{\mathrm{X}}(z)=\rho_{\mathrm{X}}(0) \mathrm{e}^{-3 w_{a} z /(1+z)}(1+z)^{3\left(1+w_{0}+w_{a}\right)}$.

For a constant $w \equiv w_{0}\left(w_{a}=0\right)$, the usual form $\rho_{\mathrm{X}}(z)=$ $\rho_{\mathrm{X}}(0)(1+z)^{3\left(1+w_{0}\right)}$ is recovered. A more general discussion of the effects of using one or more parameters for cosmological data analysis can be found in Gong \& Zhang (2005).

The comoving distance $\chi$ is defined as

$\chi(z)=\int_{0}^{z} \frac{c}{H\left(z^{\prime}\right)} \mathrm{d} z^{\prime}$, 
and the comoving angular-diameter distance $r(\chi)$ is equal, respectively, to $\chi, R_{0} \sin \left(\chi / R_{0}\right), R_{0} \sinh \left(\chi / R_{0}\right)$, for a flat, closed and open Universe where the present curvature radius of the universe is defined as $R_{0}=c /\left(\kappa H_{0}\right)$ with $\kappa^{2} \equiv 1,-\Omega_{\kappa}$, and $\Omega_{\kappa}$, respectively.

\subsection{Statistical approach}

The majority of the recent CMB analysis use Markov Chains Monte Carlo simulations (Gilks et al. 1996; Christensen \& Meyer 1998) with Bayesian inference. The philosophical debate between the Bayesian and the frequentist statistical methods is beyond the scope of this paper (for a comparison of the two approaches see, for instance, Feldman \& Cousins 1998; and Zech 2002). Here, we briefly review the principles of each method.

For a given data set, the Bayesian approach computes the probability distribution function (PDF) of the parameters describing the cosmological model. The Bayesian probability is a measure of the plausibility of an event, given incomplete knowledge. In a second step, the Bayesian constructs "credible" interval, centred near the sample mean, tempered by "prior" assumptions concerning the mean. On the other hand, the frequentist determines the probability distribution of the data as a function of the cosmological parameters and gives a confidence level that the given interval contains the parameter. In this way, the frequentist completely avoids the concept of a PDF defined for each parameter. As the questions asked by the two approaches are different, we might expect different confidence intervals. However, the philosophical difference between the two methods should not generally lead, in the end, to major differences in the determination of physical parameters and their confidence intervals when the parameters stay in a physical region.

Our work is based on the "frequentist" (or "classical") confidence level method originally defined by Neyman (1937). This avoids any potential bias due to the choice of priors.

\subsubsection{Confidence levels with a frequentist approach}

For a given cosmological model defined by the $n$ cosmological parameters $\theta=\left(\theta_{1}, \ldots, \theta_{n}\right)$, and for a data set of $N$ quantities $x=\left(x_{1}, \ldots, x_{N}\right)$ measured with Gaussian experimental errors $\sigma_{x}=\left(\sigma_{1}, \ldots, \sigma_{N}\right)$, neglecting the correlations between the $N$ measurements $x_{i}$, the likelihood function can be written as:

$\mathcal{L}\left(x, \sigma_{x} ; \theta\right)=\frac{1}{\sqrt{2 \pi} \sigma_{i}} \exp \left(-\frac{\left(x_{i}-x_{i, \text { model }}\right)^{2}}{2 \sigma_{i}^{2}}\right)$,

where $x_{\text {model }}=\left(x_{1, \text { model }}, \ldots, x_{N, \text { model }}\right)$ is a set of corresponding model-dependent values.

In the rest of this paper, we adopt a $\chi^{2}$ notation, which means that the following quantity is minimized:

$\chi^{2}\left(x, \sigma_{x} ; \theta\right)=-2 \ln \left(\mathcal{L}\left(x, \sigma_{x} ; \theta\right)\right)$.

We first determine the minimum $\chi_{0}^{2}$ of $\chi^{2}\left(x, \sigma_{x} ; \theta\right)$ having let all the cosmological parameters free. Then, to set a confidence level (CL) on any individual cosmological parameter $\theta_{i}$, we scan the variable $\theta_{i}$ : for each fixed value of $\theta_{i}$, we again minimize $\chi^{2}\left(x, \sigma_{x} ; \theta\right)$ but with $n-1$ free parameters. The $\chi^{2}$ difference, $\Delta \chi^{2}\left(\theta_{i}\right)$, between the new minimum and $\chi_{0}^{2}$, allows us to compute the $\mathrm{CL}$ on the variable, assuming that the experimental errors are Gaussian,

$$
1-\operatorname{CL}\left(\theta_{i}\right)=\frac{1}{\sqrt{2^{N_{\text {d.o.f. }}} \Gamma\left(N_{\text {d.o.f. }} / 2\right)}} \int_{\Delta \chi^{2}\left(\theta_{i}\right)}^{\infty} \mathrm{e}^{-t / 2} t^{N_{\text {d.o.f. }} / 2-1} \mathrm{~d} t
$$

where $\Gamma$ is the gamma function and the number of degrees of freedom $N_{\text {d.o.f. }}$ is equal to 1 . This method can be easily extended to two variables. In this case, the minimizations are performed for $n-2$ free parameters, and the confidence level $\mathrm{CL}\left(\theta_{i}, \theta_{j}\right)$ is derived from Eq. (8) with $N_{\text {d.o.f. }}=2$.

By definition, this frequentist approach does not require any marginalization to determine the sensitivity on a single individual cosmological parameter. Moreover, in contrast to Bayesian treatment, no prior on the cosmological parameters is needed. With this approach, the correlations between the variables are naturally taken into account and the minimization fit can explore the whole phase space of the cosmological parameters.

In this study, the minimizations of $\chi^{2}\left(x, \sigma_{x} ; \theta\right)$ are performed with the MINUIT package (James 1978). For the 9-parameter study proposed in this paper, each fit requires around 200 calculations of $\chi^{2}$. The consumed CPU-time is dominated by the computation of the angular power spectrum $\left(C_{\ell}\right)$ of the CMB in CMBEASY (Doran 2003). In practice, to get the CL for one variable, as shown, for instance, in Fig. 1, the computation of the $C_{\ell}$ is done around 10000 times. This method is very powerful for studying the impacts of the parameters: it is not costly to add or remove parameters because the number of $C_{\ell}$ computations scales with the number of parameters.

\subsubsection{Combination of cosmological probes with Fisher matrices}

In parallel with this frequentist approach, to study the statistical sensitivities of different combinations of future surveys, we perform a prospective analysis based on the Fisher matrix technique (Fisher 1935). We check that the Fisher approximation gives similar results to the global frequentist method described above.

The statistical errors on the $n$ cosmological parameters $\theta=\left(\theta_{1}, \ldots, \theta_{n}\right)$ are determined by using the inverse of the covariance matrix $V$ called the Fisher matrix $F$ defined as:

$\left(V^{-1}\right)_{i j}=F_{i j}=-\frac{\partial^{2} \ln \mathcal{L}(x ; \theta)}{\partial \theta_{i} \partial \theta_{j}}$,

where $\mathcal{L}(x ; \theta)$ is the likelihood function depending on the $n$ cosmological parameters and a data set of $N$ measured quantities $x=\left(x_{1}, \ldots, x_{N}\right)$. A lower bound, and often a good estimate, for the statistical error on the cosmological parameter $\theta_{i}$ is given by $\left(V_{i i}\right)^{1 / 2}$.

When the measurements of several cosmological probes are combined, the total Fisher matrix $F_{\text {tot }}$ is the sum of the three Fisher matrices $F_{\mathrm{SN}}, F_{\mathrm{WL}}$ and $F_{\mathrm{CMB}}$ corresponding respectively to the SNIa, weak lensing, and CMB observations. 
The total covariance matrix $F_{\text {tot }}^{-1}$ allows us to estimate both (i) the expected sensitivity on the cosmological parameters, with the diagonal terms, and (ii) the correlations between the parameters, with the off-diagonal terms. The Fisher matrices for each probe are computed as follows.

CMB: In the case of CMB experiments, the data set vector $x$, defined in Eq. (6), corresponds to the measurements of $C_{\ell}$, the angular power spectrum of the CMB from $\ell=2$ to some cutoff $\ell_{\text {max }}$. If the likelihood of each individual $C_{\ell}$ is assumed to be Gaussian with dispersion $\sigma_{C_{\ell}}$, Eq. (9) simplifies to:

$\left(F_{\mathrm{CMB}}\right)_{i j}=\sum_{l=2}^{\ell_{\max }} \frac{1}{\sigma_{C_{\ell}}^{2}} \cdot \frac{\partial C_{\ell}}{\partial \theta_{i}} \cdot \frac{\partial C_{\ell}}{\partial \theta_{j}}$

where $\sigma_{C_{\ell}}$ is the statistical error on $C_{\ell}$ obtained directly from published results or estimated as (see Knox 1995):

$\sigma_{C_{\ell}}=\sqrt{\frac{2}{(2 \ell+1) f_{\text {sky }}}}\left[C_{\ell}+\left(\theta_{f w h m} s\right)^{2} \cdot \mathrm{e}^{\frac{\ell^{2} \theta_{f w h m}^{2}}{8 \ln (2)}}\right]$

where the second term incorporates the effects of instrumental noise and beam smearing. In Eq. (11), $\theta_{f w h m}, f_{\text {sky }}$, and $s$ are the angular resolution, the fraction of the sky observed and the expected sensitivity per pixel,respectively.

The $C_{\ell}$ and their derivatives with respect to the various cosmological parameters are computed with CMBEASY (Doran 2003), an object-oriented $\mathrm{C}++$ package derived from CMBFAST (Seljak \& Zaldarriaga 1996).

SNIa: The SNIa apparent magnitudes $m$ can be expressed as a function of the luminosity distance as

$$
\begin{aligned}
m(z)=M+\mu(z) & =M_{s_{0}}+5 \log _{10}\left(H_{0}\right)-25+\mu(z) \\
& =M_{s_{0}}+5 \log _{10}\left(D_{L}\right)
\end{aligned}
$$

where $M$ is the absolute magnitude of SNIa, $M_{s_{0}}$ may be considered as a normalization parameter and $D_{L}(z) \equiv H_{0} d_{L}(z)$ is the $H_{0}$-independent luminosity distance to an object at redshift $z$. Then $\mu$ are the distance moduli given in Riess et al. (2004) with definition $\mu=5 \log _{10}\left(d_{L}\right)+25$. We can fit either $\mu(z)$ or $m(z)$ by changing the normalization parameter. It is $M_{s_{0}}$ or $M_{s_{0}}-M$ when $m(z)$ or $\mu(z)$ are fitted, respectively. We emphasize that this change of definition has no effect on our results as the normalization parameter has no physical meaning in this analysis. The usual luminosity distance $d_{L}(z)$ is related to the comoving angular-diameter distance $r(\chi)$ by $d_{L}(z)=(1+z) \cdot r(\chi)$, with the definition of $r(\chi)$ and $\chi(z)$ given in Sect. 2.1.

In this case, the $x_{i}$ in Eq. (6) correspond to the magnitude or distance moduli. Therefore the Fisher matrix is related to the measured apparent magnitude Gaussian $m_{k}$ of each object and its statistical errors $\sigma_{m_{k}}$ by

$$
\left(F_{\mathrm{SN}}\right)_{i j}=\sum_{k} \frac{1}{\sigma_{m_{k}}^{2}} \cdot \frac{\partial m_{k}}{\partial \theta_{i}} \cdot \frac{\partial m_{k}}{\partial \theta_{j}}
$$

Weak lensing: The weak lensing power spectrum is given by (e.g., Hu \& Tegmark 1999, cf., Refregier 2003 for conventions)

$C_{\ell}=\frac{9}{16}\left(\frac{H_{0}}{c}\right)^{4} \Omega_{\mathrm{m}}^{2} \int_{0}^{\chi \mathrm{h}} \mathrm{d} \chi\left[\frac{g(\chi)}{\operatorname{ar}(\chi)}\right]^{2} P\left(\frac{\ell}{r}, \chi\right)$,

where $r(\chi)$ is the comoving angular-diameter distance, and $\chi_{\mathrm{h}}$ corresponds to the comoving distance to horizon. The radial weight function $g$ is given by

$g(\chi)=2 \int_{\chi}^{\chi_{\mathrm{h}}} \mathrm{d} \chi^{\prime} n\left(\chi^{\prime}\right) \frac{r(\chi) r\left(\chi^{\prime}-\chi\right)}{r\left(\chi^{\prime}\right)}$,

where $n(\chi)$ is the probability of finding a galaxy at comoving distance $\chi$ and is normalized as $\int \mathrm{d} \chi n(\chi)=1$.

The linear matter power spectrum $P(k, z)$ is computed using the transfer function from Bardeen et al. (1986) with the conventions of Peacock (1997), thus ignoring the corrections on large scales for quintessence models (Ma et al. 1999). The linear growth factor of the matter over-densities $\delta$ is given by the well known equation:

$\ddot{\delta}+2 H \dot{\delta}-\frac{3}{2} H^{2} \Omega_{\mathrm{m}}(a) \delta=0$,

where dots correspond to time derivatives, and $\Omega_{\mathrm{m}}(a)$ is the matter density parameter at the epoch corresponding to the dimensionless scale factor $a$. This equation is integrated numerically with boundary conditions given by the matter-dominated solution, $G=\delta / a=1$ and $\dot{G}=0$, as $a \rightarrow 0$ (see e.g. Linder \& Jenkins 2003). We enforce the CMB normalization of the power spectrum $P(k, 0)$ at $z=0$ using the relationship between the WMAP normalization parameter $A$ and $\sigma_{8}$ given by $\mathrm{Hu}$ (2004). Considerable uncertainties remain for the nonlinear corrections in quintessence models (cf. discussion in $\mathrm{Hu}$ 2002). Here, we use the fitting formula from Peacock \& Dodds (1996).

For a measurement of the power spectrum, the Fisher matrix element is defined as:

$\left(F_{\mathrm{WL}}\right)_{i j}=\sum_{\ell} \frac{1}{\sigma_{C_{\ell}}^{2}} \frac{\partial C_{\ell}}{\partial \theta_{i}} \frac{\partial C_{\ell}}{\partial \theta_{j}}$

where the summation is over modes $\ell$, which can be reliably measured. This expression assumes that the errors $\sigma_{C_{\ell}}$ on the lensing power spectrum are Gaussian and that the different modes are uncorrelated. Mode-to-mode correlations have been shown to increase the errors on cosmological parameters (Cooray \& Hu 2001) but are neglected in this paper.

Neglecting non-Gaussian corrections, the statistical error $\sigma_{C_{\ell}}$ in measuring the lensing power spectrum $C_{\ell}$ (cf., e.g., Kaiser 1998; Hu \& Tegmark 1999; Hu 2002) is given by:

$\sigma_{C_{\ell}}=\sqrt{\frac{2}{(2 l+1) f_{\text {sky }}}}\left(C_{l}+\frac{\sigma_{\gamma}^{2}}{2 n_{\mathrm{g}}}\right)$,

where $f_{\text {sky }}$ is the fraction of the sky covered by the survey, $n_{\mathrm{g}}$ the surface density of usable galaxies, and $\sigma_{\gamma}^{2}=\left\langle|\gamma|^{2}\right\rangle$ is the shear variance per galaxy arising from intrinsic shapes and measurement errors. 


\subsection{Cosmological parameters and models}

For the studies presented in this paper, we limit ourselves to the 9 cosmological parameters: $\theta=\Omega_{\mathrm{b}}, \Omega_{\mathrm{m}}, h, n_{s}, \tau, w_{0}, w_{a}, A$, and $M_{s_{0}}$, with the following standard definitions:

- $\left(\Omega_{i}, i=b, m\right)$ are densities for baryon and matter, respectively ( $\Omega_{\mathrm{m}}$ includes both dark matter and baryons);

$-h$ is the Hubble constant in units of $100 \mathrm{~km} \mathrm{~s}^{-1} \mathrm{Mpc}^{-1}$;

$-n_{s}$ is the spectral index of the primordial power spectrum;

$-\tau$ is the reionization optical depth;

- $A$ is the normalization parameter of the power spectrum for CMB and weak lensing (cf. Hu \& Tegmark (1999) for definitions). The matter power spectrum is normalized according to the COBE normalization (Bunn \& White 1997). This is consistent with the WMAP results (Spergel et al. 2003) and with the average of recent cosmic shear measurements (see compilation tables in Mellier et al. 2002; Hoekstra et al. 2002; Refregier 2003);

- $M_{s_{0}}$ is the normalization parameter from SNIa (cf. Sect. 2.2.2);

- Dark energy is described by the $w_{0}$ parameter corresponding to the value of the equation of state at $z=0$. When the $z$ dependence of the equation of state is studied, an additional parameter $w_{a}$ is defined (cf. Sect. 2.1).

The reference fiducial model of our simulation is a $\Lambda \mathrm{CDM}$ model with parameters $\Omega_{\mathrm{m}}=0.27, \Omega_{\mathrm{b}}=0.0463, n_{s}=0.99$, $h=0.72, \tau=0.066, A=0.86\left(\sigma_{8}=0.88\right)$, consistent with the WMAP experiment (see Tables 1-2 in Spergel et al. 2003). In agreement with this experiment, we assume throughout this paper that the universe is flat, i.e., $\Omega=\Omega_{\mathrm{m}}+\Omega_{\mathrm{X}}=1$. We also neglect the effect of neutrinos, using 3 degenerate families of neutrinos with masses fixed to 0 .

In the following, we will consider deviations from this reference model. For the equation of state, we use $w_{0}=-0.95$ and $w_{a}=0$ as central reference values (we have not used the exact $w_{0}=-1$ value to avoid transition problems in the CMB calculations). To estimate the sensitivity on the parameters describing the equation of state, we also consider two other fiducial models: a SUGRA model (Brax \& Martin 1999), with $\left(w_{0}=-0.8, w_{a}=0.3\right)$ as proposed by, e.g., Weller \& Albrecht (2002) to represent quintessence models, and a phantom model (Caldwell 2002) with $\left(w_{0}=-1.2, w_{a}=-0.3\right)$.

In this analysis, the full covariance matrix on all parameters is used with no prior constraints on the parameters, avoiding biases from internal degeneracies. We have implemented the time evolving parameterization of the equation of state in simulations and analysis of the three probes we consider in this paper, i.e. CMB, SNIa, and weak lensing.

\section{Combination of current surveys}

We first apply our statistical approach to the combination of recent SNIa and CMB data, without any external constraints or priors, and then compare our results with other published results. The importance of introducing dark energy perturbations has already been discussed by Weller \& Lewis (2003). However, the nature of the dark energy is unknown, and it is possible that it is homogeneous and there is no clustering as, for example, in some modified gravity models. Some authors suggest to remove dark energy clustering from the CMB code (see for instance Tegmark et al. 2004). Therefore, in this section, we first compare the effect of switching the dark energy perturbations on and off for $w>-1$, in the case of current SNIa and CMB surveys. We then compare the statistical errors obtained with a global fit using this frequentist treatment (without dark energy perturbations), with those predicted by the Fisher matrix technique.

\subsection{Current surveys}

We use the "Gold sample" data compiled by Riess et al. (2004), with 157 SNIa including a few at $z>1.3$ from the Hubble Space Telescope (HST GOODS ACS Treasury survey), and the published data from WMAP taken from Hinshaw et al. (2003). For the latter, we neglect the correlations between the $C_{\ell}$ measurements and assume that the errors follow a Gaussian law. We check that these assumptions do not alter our conclusions by considering the full likelihood provided by the WMAP team (Verde et al. 2003) and by comparing the $\chi^{2}$ distributions for the full likelihood and for the simplified likelihood of Eq. (6). We conclude that both the central value and the error of the fitted cosmological parameters are not affected by this simplification.

We performed two distinct analyses: in the first case, the equation of state is held constant with a single parameter $w_{0}$ and we fit 8 parameters, as described in Sect. 2.2; in the second case, the $z$-dependence of the equation of state is modelled by two variables $w_{0}$ and $w_{a}$ as defined in Sect. 2.1, and we fit 9 parameters.

\subsection{Results}

First, we do not consider any perturbation for the dark energy. The results of our frequentist combination of CMB and SNIa data are summarized in Table 1. When the equation of state is considered constant, we obtain $w_{0}=-0.92_{-0.13}^{+0.10+0.20}$ at $68 \% \mathrm{CL}(1 \sigma)$ and $95 \% \mathrm{CL}(2 \sigma)$. The shape of the CL is relatively symmetrical around the value of $w_{0}$ obtained at the $\chi 2$ minimum. When a $z$ dependence is added to the equation of state, the CL is still symmetrical with $w_{0}=-1.09_{-0.15}^{+0.13+0.25}$ but the $w_{a}$ distribution becomes asymmetrical with a long tail for lower values of $w_{a}$, as can be seen in Fig. 1. The 1-D CL for $w_{a}$ gives the resulting CL: $w_{a}=0.82_{-0.26-0.80}^{+0.21}$.

With our choice of parameterization (see Eq. (3)), we get the boundary condition $w_{0}+w_{a}<0$. When a fit solution is found close to this limit, as is the case with the current data, the CL distributions are asymmetric, giving asymmetrical errors like those shown in Fig. 2. The errors on the parameters are strongly dependent on the central values of the various cosmological parameters. For example, the error on $w_{a}$ increases when the $\left(w_{0}, w_{a}\right)$ solution moves away from the "unphysical" region $w_{0}+w_{a}>0$.

Our results can be compared with those of Seljak et al. (2004), who have also removed dark energy perturbations. 
Table 1. Results of the frequentist fit to WMAP and Riess et al. (2004) SNIa data assuming no perturbation in dark energy. For the 8-parameter fit with a constant EOS, the first column gives the value of the variable at the $\chi^{2}$ minimum, with the confidence interval at $68 \%(1 \sigma)$, and the second column shows the $1 \sigma$ error computed with the Fisher matrix techniques. The third and fourth columns present the same information for the 9-parameter fit with a $z$-dependent EOS. Note the $w_{a}$ error is very asymmetrical and goes from ${ }_{-0.26}^{+0.21}$ for CL at $68 \%$ to ${ }_{-0.80}^{+0.42}$ for CL at $95 \%$ (see text).

\begin{tabular}{lcc|cc}
\hline \hline & \multicolumn{2}{c|}{ Constant EOS } & \multicolumn{2}{c}{$z$ dependent EOS } \\
& fit & $\sigma_{\text {Fisher }}$ & fit & $\sigma_{\text {Fisher }}$ \\
\hline$\Omega_{\mathrm{b}}$ & $0.049_{-0.003}^{+0.005}$ & \pm 0.003 & $0.055_{-0.003}^{+0.003}$ & \pm 0.003 \\
$\Omega_{\mathrm{m}}$ & $0.29_{-0.04}^{+0.05}$ & \pm 0.04 & $0.33_{-0.04}^{+0.04}$ & \pm 0.04 \\
$h$ & $0.69_{-0.02}^{+0.03}$ & \pm 0.03 & $0.69_{-0.02}^{+0.03}$ & \pm 0.03 \\
$n_{S}$ & $0.97_{-0.03}^{+0.03}$ & \pm 0.03 & $0.97_{-0.03}^{+0.03}$ & \pm 0.03 \\
$\tau$ & $0.13_{-0.04}^{+0.04}$ & \pm 0.04 & $0.14_{-0.04}^{+0.04}$ & \pm 0.04 \\
$w_{0}$ & $-0.92_{-0.13}^{+0.10}$ & \pm 0.11 & $-1.09_{-0.15}^{+0.13}$ & \pm 0.14 \\
$w_{a}$ & - & - & $0.82_{-0.26}^{+0.21}$ & \pm 0.25 \\
$A$ & $0.79_{-0.07}^{+0.08}$ & \pm 0.10 & $0.80_{-0.07}^{+0.08}$ & \pm 0.10 \\
$M_{s 0}$ & $15.94_{-0.03}^{+0.03}$ & \pm 0.03 & $15.95_{-0.03}^{+0.03}$ & \pm 0.03 \\
\hline
\end{tabular}
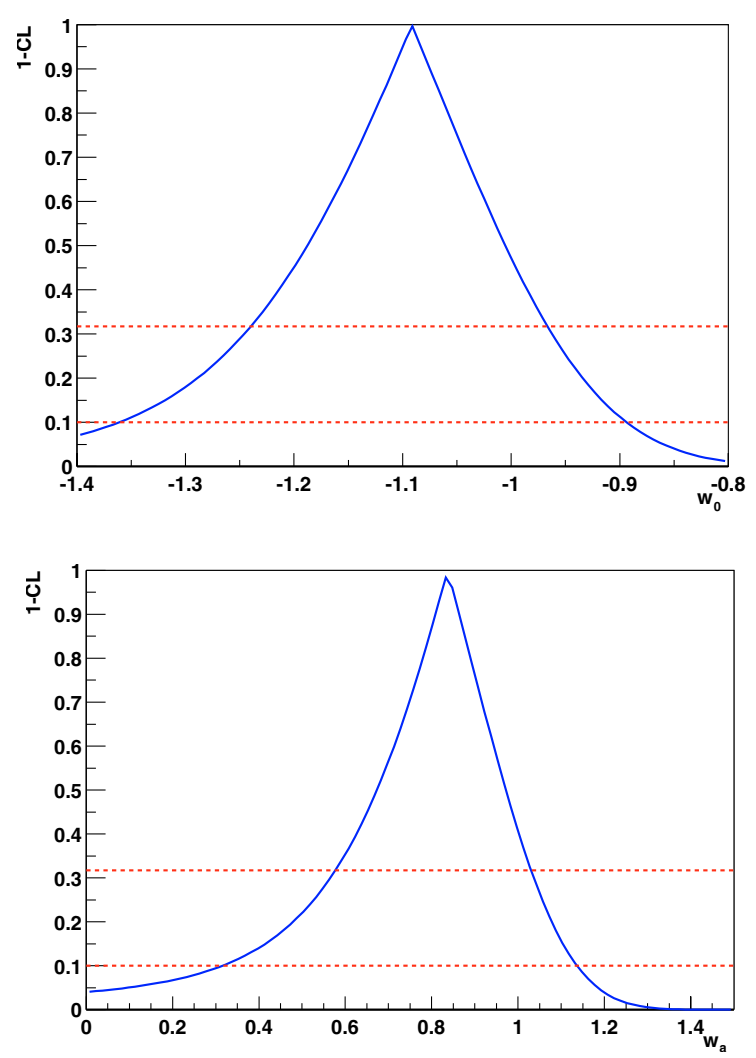

Fig. 1. Confidence level (CL) plots on parameters $w_{0}$ (top) and $w_{a}$ (bottom), using WMAP and Riess et al. (2004) SNIa data for a 9-parameter fit with evolving EOS. The dashed lines correspond to the $68 \%(1 \sigma)$ and $90 \%(1.64 \sigma)$ confidence intervals.

Their central value corresponds to $w_{0}=-0.98_{-0.37}^{+0.38}$ and $w_{a}=$ $-0.05_{-1.13}^{+1.92}$ at $95 \%(2 \sigma)$. These results are closer to $w=-1$ than ours and give larger errors for $w_{a}$ than the ones we get, because their solution is far from the "unphysical" region. However,
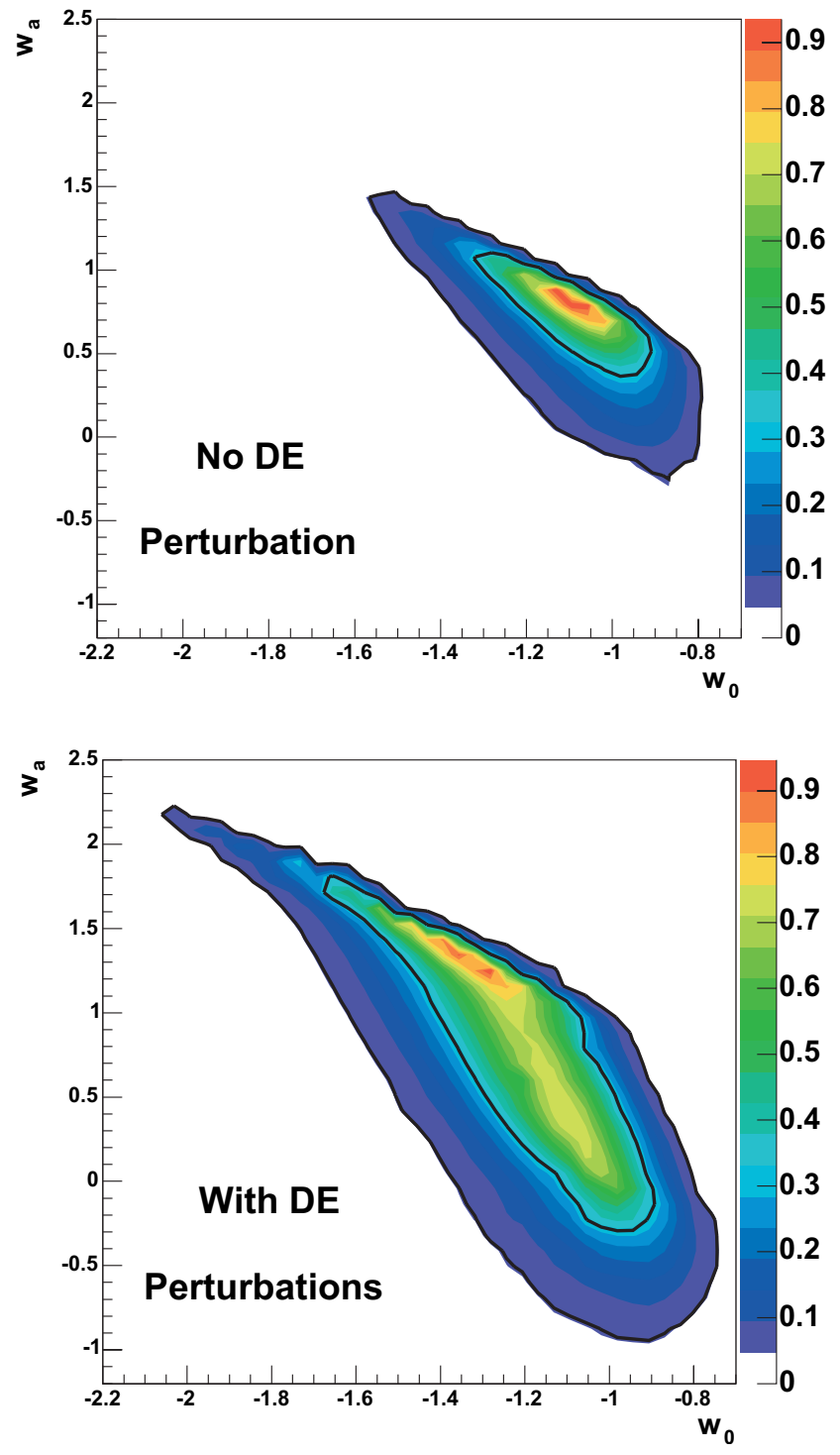

Fig. 2. Confidence level contour plots with WMAP and Riess et al. (2004) SNIa data, for the 9-parameter fit with a $z$-dependent EOS in the plane $\left(w_{0}, w_{a}\right)$. The top plot corresponds to the case when we introduce no dark energy perturbation. For the bottom plot, we introduce dark energy perturbations only when $w>-1$. The solid lines correspond to $68 \%(1 \sigma)$ and $95 \%(2 \sigma) \mathrm{CL}$.

the comparison is not exact, since they use a Bayesian approach for the fits, and only give results for an evolving equation of state, for the total combination of the WMAP and SNIa data with other SDSS probes (galaxies clustering, bias, and Lyman $\alpha$ forest).

We also perform the fits, including dark energy perturbations, only when $w>-1$ (which is the default implementation in CMBFAST). Caldwell \& Doran (2005) argue convincingly that crossing the cosmological constant boundary leaves no distinct imprint; i.e., the contributions of $w<-1$ are negligible, because $w<-1$ dominates only at late times and dark energy does not generally give strong gravitational clustering. Our analysis, including dark energy perturbations only when $w>-1$, gives a minimum (cf. right hand side plot in Fig. 2) for $w_{0}=-1.32_{-0.19-0.45}^{+0.15+0.30}$ and $w_{a}=1.2_{-0.8-1.7}^{+0.5+1.0}$ with CL at $68 \%$ 
and $95 \%$. This is some $2 \sigma$ away from the no perturbation case. These values are very close to those obtained by Upadhye et al. (2004), who use a procedure similar to ours, without any marginalization on parameters, a weak constraint $w_{0}+w_{a} \leq 0$ inside their fit. Their result, $w_{0}=-1.3_{-0.39}^{+0.34}$ and $w_{a}=1.25_{-2.17}^{+0.40}$ at $95 \%(2 \sigma)$, almost has the same central value as our fit, when we switch the dark energy perturbation for $w>-1$ on. The errors we get are also compatible, and are much larger than in the no perturbation case. Weller \& Lewis (2003) combined WMAP and SNIa analysis with a constant sound speed and also report a more negative value of $w$, when a redshift dependence is taken into account. Although Rapetti et al. (2004) observe a reduced effect when they add cluster data, they still indicate a similar trend. Finally, when dark energy perturbations are included, we observe that the minimization is more difficult and correlations between parameters increase.

We conclude that our results are compatible with other published papers using various combinations of cosmological probes. There is good agreement of all analysis when $w_{0}$ is constant, showing that data agree well with the $\Lambda$ CDM model. However, large uncertainties remain for the location of the minimum in the $\left(w_{0}, w_{a}\right)$ plane, when a redshift variation is allowed. We emphasize that this is not due to the statistical method but to internal assumptions. Upadhye et al. (2004) mention the sensitivity to the choice of parameterization. We show that the introduction of dark energy perturbations for $w>-1$, can change the minimum by nearly $2 \sigma$ and that the minimum is not established well, as correlations between parameters increase, and errors in this zone of parameter space are very large.

For the rest of the study, we have decided to consider only one of the two options for the dark energy perturbations. We switched the dark energy perturbations off, since the dark energy nature is not known and there could be no clustering and since it is technically the simpler solution. As dark energy perturbations for $w>-1$ degrade the errors, our approach may provide optimistic results. What we can underline is not the absolute error determination, but the comparison between different probe combinations.

Finally, in the case without dark energy perturbation, Table 1 compares the $1 \sigma$ errors obtained with the frequentist method and the errors predicted with the Fisher matrix techniques. The agreement is good. In the remaining part of this paper, we will use the Fisher matrix approach for the combination of expectations from future surveys, with fiducial values of $w_{a}$ close to zero to avoid the problem of physical boundary discussed previously.

\section{Combination of future surveys}

Many studies have combined CMB and SNIa for varying $w$, but few have studied the effect of a combination with multiple $z$ weak lensing analysis, in detail and coherently. In this section, we present the sensitivity of the combination of future CMB, SNIa, and weak lensing surveys for dark energy evolution. We expect new measurements from the CHTLS surveys in SNIa and weak lensing in the next few years, which can be combined with the first-year WMAP, together with the expected
Table 2. Number of simulated SNIa by bins of 0.1 in redshift for midterm scenario (SNLS+HST) and long-term scenario (SNAP).

\begin{tabular}{l|cccccccc}
\hline \hline$z$ & 0.2 & 0.3 & 0.4 & 0.5 & 0.6 & 0.7 & 0.8 & 0.9 \\
\hline Mid-term & - & 44 & 56 & 80 & 96 & 100 & 104 & 108 \\
Long-term & 35 & 64 & 95 & 124 & 150 & 171 & 183 & 179 \\
\hline$z$ & 1.0 & 1.1 & 1.2 & 1.3 & 1.4 & 1.5 & 1.6 & 1.7 \\
\hline Mid-term & 10 & 14 & 7 & 12 & 5 & 2 & 3 & 1 \\
Long-term & 170 & 155 & 142 & 130 & 119 & 107 & 94 & 80 \\
\hline
\end{tabular}

CMB data from the Olimpo CMB balloon experiment. These are what we call "mid-term" surveys.

The combined mid-term results will be compared to the "long-term" expectations from the next generation of observations in space that are under preparation, i.e., the Planck Surveyor mission for CMB expected in 2007 and the SNAP/JDEM mission, a large imaging survey expected for 2014, which includes both SNIa and weak lensing surveys.

\subsection{Mid-term surveys}

The different assumptions we use for the mid-term simulations are as follows.

CMB: We add to the WMAP data some simulated CMB expectations from the Olimpo balloon experiment (Masi et al. 2003) equipped with a $2.6 \mathrm{~m}$ telescope and 4 bolometers arrays for frequency bands centred at 143, 220, 410, and $540 \mathrm{GHz}$. This experiment will also allow us to observe the first "large" survey of galaxies cluster through the SZ effect. For this paper, we will limit our study to CMB anisotropy aspects.

For a nominal 10-day flight with an angular resolution of $\theta_{\text {fwhm }}=4^{\prime}$ and with $f_{\text {sky }} \simeq 1 \%$, the expected sensitivity per pixel is $s=3.4 \times 10^{-6}$. We use Eq. (11) to estimate the statistical error $\sigma_{C_{\ell}}$ on the angular power spectrum.

SNIa: We simulate future SNIa measurements derived from the large SNLS (2001) ground-based survey within the CFHTLS (2001). This survey started in 2003 and expects to collect a sample of 700 identified SNIa in the redshift range $0.3<z<1$, after 5 years of observations. We simulate the sample, as explained in Virey et al. (2004a) with the number of SNIa shown in Table 2, in agreement with the expected SNIa rates from SNLS. We assume a magnitude dispersion of 0.15 for each supernova, constant in redshift after all corrections. This uncertainty corresponds to the most favorable case in which experimental systematic errors are not considered. It is the dominating factor in the systematical errors.

A set of 200 very well-calibrated SNIa at redshift $<0.1$ should be measured by the SN factory (Wood-Vasey et al. 2004) project. This sample is needed to normalize the Hubble diagram and will be called the "nearby" sample.

Finally, to be as complete as possible, we simulate a set of 54 SNIa, expected from HST programs, with a magnitude dispersion of 0.17 for each supernova, at redshifts between 1 and 1.7. Table 3 summarizes the simulation parameters. 
Table 3. Simulation inputs for CMB, SNIa and Weak Lensing observations.

\begin{tabular}{|c|c|c|c|c|c|}
\hline \multicolumn{6}{|c|}{ CMB surveys } \\
\hline $\begin{array}{l}\text { Current } \\
\text { Data }\end{array}$ & WMAP (Spergel et al. 2003) & $\begin{array}{c}f_{\text {sky }} \\
\text { full sky }\end{array}$ & $\begin{array}{c}f(\mathrm{GHz}) \\
23 / 33 / 41 / 61 / 94\end{array}$ & $\begin{array}{c}\theta_{f w h m}(\prime) \\
13\end{array}$ & $\begin{array}{c}s\left(10^{-6}\right) \\
-\end{array}$ \\
\hline $\begin{array}{l}\text { Mid-term } \\
\text { Data }\end{array}$ & $\begin{array}{l}\text { Olimpo } \\
+ \text { WMAP }\end{array}$ & 0.01 & $143 / 220 / 410 / 540$ & 4 & 3.4 \\
\hline $\begin{array}{l}\text { Long-term } \\
\text { data }\end{array}$ & Planck & full Sky & $\begin{array}{l}100 \\
143 \\
217\end{array}$ & $\begin{array}{l}9.2 \\
7.1 \\
5.0\end{array}$ & $\begin{array}{l}2.0 \\
2.2 \\
4.8\end{array}$ \\
\hline \multicolumn{6}{|c|}{ SN surveys } \\
\hline $\begin{array}{l}\text { Current } \\
\text { Data }\end{array}$ & Riess et al. (2004) + HST & $\begin{array}{c}\text { SN \# } \\
157\end{array}$ & $\begin{array}{c}\text { Redshift range } \\
z<1.7\end{array}$ & $\begin{array}{c}\text { Statistical error } \\
\sim 0.25\end{array}$ & $\begin{array}{c}\text { Systematic error } \\
-\end{array}$ \\
\hline $\begin{array}{l}\text { Mid-term } \\
\text { Data }\end{array}$ & $\begin{array}{c}\text { SNfactory } \\
\text { SNLS } \\
\text { HST }\end{array}$ & $\begin{array}{c}200 \\
700 \\
54\end{array}$ & $\begin{aligned} z & <0.1 \\
0.3 & <z<1 \\
1 & <z\end{aligned}$ & $\begin{array}{l}0.15 \\
0.15 \\
0.17\end{array}$ & - \\
\hline $\begin{array}{l}\text { Long-term } \\
\text { Data }\end{array}$ & $\begin{array}{l}\text { SNfactory } \\
\text { SNAP }\end{array}$ & $\begin{array}{c}300 \\
2000\end{array}$ & $\begin{array}{c}z<0.1 \\
0.1<z<1.7\end{array}$ & $\begin{array}{l}0.15 \\
0.15\end{array}$ & 0.02 \\
\hline \multicolumn{6}{|c|}{ WL surveys } \\
\hline $\begin{array}{l}\text { Mid-term } \\
\text { Data }\end{array}$ & CFHTLS & $\begin{array}{c}z_{\mathrm{m}}(2 \text { bins }) \\
0.72,1.08\end{array}$ & $\begin{array}{c}A\left(\mathrm{deg}^{2}\right) \\
170\end{array}$ & $\begin{array}{c}\text { total } n_{\mathrm{g}}\left(\operatorname{arcmin}^{-2}\right) \\
20\end{array}$ & $\begin{array}{c}\sigma_{\gamma} \\
0.35\end{array}$ \\
\hline $\begin{array}{l}\text { Long-term } \\
\text { Data }\end{array}$ & SNAP & $0.95,1.74$ & 1000 & 100 & 0.31 \\
\hline
\end{tabular}

Weak lensing: The coherent distortions that lensing induces on the shape of background galaxies have now been firmly measured from the ground and from space. The amplitude and angular dependence of this "cosmic shear" signal can be used to set strong constraints on cosmological parameters.

Earlier studies of the constraints on dark energy from generic weak lensing surveys can be found in $\mathrm{Hu} \&$ Tegmark (1999), Huterer (2001), and Hu (2002). More recently, predictions of the constraints on an evolving $w(a)$ were studied by several authors (e.g., Benabed \& van Waerbeke 2004; Lewis \& Bridle 2002).

In this paper, we consider measurements of the lensing power spectrum $C_{\ell}$ with galaxies in two redshift bins. We will only consider modes between $\ell=10$ and 20000 , thus avoiding small scales where instrumental systematics and theoretical uncertainties are more important.

We expect new cosmic shear results from the CFHTLS wide survey (CFHTLS 2001) in the near future. A sky coverage of $170^{\circ 2}$ is assumed. The rms shear error per galaxy is taken as $\sigma_{\gamma}=0.35$ and the surface density of usable galaxies as $20 \mathrm{arcmin}^{-2}$, which is divided evenly into two redshift bins with median redshifts $z_{\mathrm{m}}=0.72$ and 1.08. The redshift distribution of the galaxies in each redshift bin is taken as in Bacon et al. (2000) with the above median redshifts (cf. Table 3 for a summary of the survey parameters). We then use Eq. (18) to estimate the statistical error $\sigma_{C_{\ell}}$.

\subsection{Long-term surveys}

The future will see larger surveys both from the ground and space. To estimate the gain for large ground surveys compared to space experiments, critical studies which take the intrinsic ground limitation (both in distance and in systematics) into account, should be done, and systematic effects, not included here, will be the dominant limitation. In this paper, we limit ourselves to the future space missions.

We simulate the Planck Surveyor mission using Eq. (11) with the performances described in Tauber et al. (2004). Assuming that the other frequency bands will be used to identify the astrophysical foregrounds for the CMB study over the whole sky, we consider only the three frequency bands $(100,143$, and $217 \mathrm{GHz})$ with a resolution $\left(\theta_{\text {fwhm }}=\right.$ $9.2^{\prime}, 7.1^{\prime}$, and $5.0^{\prime}$, respectively) and a sensitivity per pixel of $\left(s=2.0 \times 10^{-6}, 2.2 \times 10^{-6}\right.$, and $\left.4.8 \times 10^{-6}\right)$.

We also simulate observations from the future SNAP satellite, a $2 \mathrm{~m}$ telescope that plans to discover around 2000 identified SNIa at redshift $0.2<z<1.7$ with very precise photometry and spectroscopy. The SNIa distribution, given in Table 2, is taken from Kim et al. (2004). The magnitude dispersion $\sigma(m)_{\text {disp }}$ is assumed to be 0.15 , constant and independent of the redshift, for all SNIa after correction. Moreover, we introduce an irreducible systematic error $\sigma(m)_{\text {irr }}$ following the prescription of Kim et al. (2004). In consequence, the total error on the magnitude $\sigma(m)_{\text {tot }}$ per redshift bin $i$ is defined 

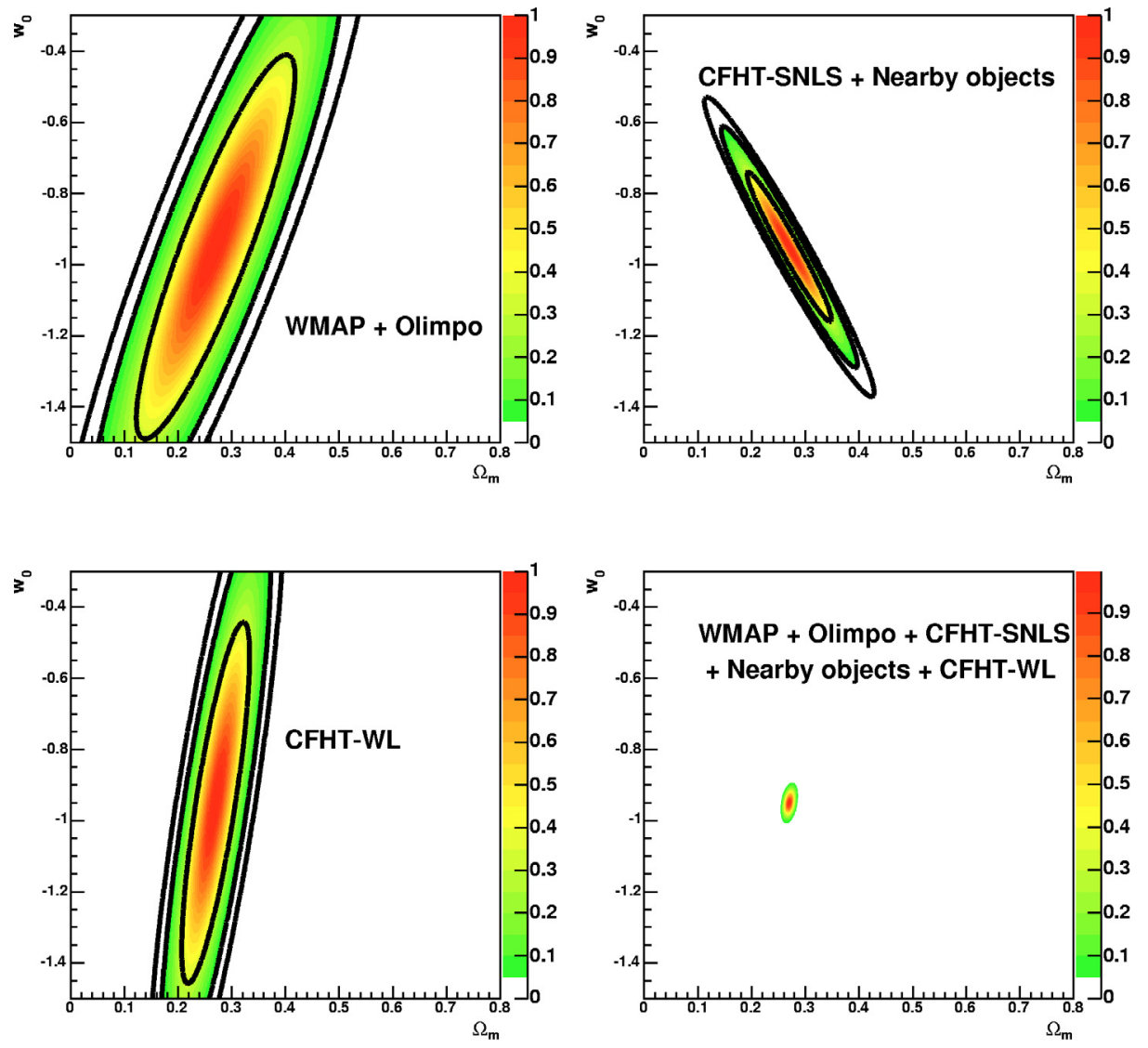

Fig. 3. The CL contours for mid-term CMB (WMAP+Olimpo), SNIa, and weak lensing data from CFHTLS and the combination of the three probes for the 8-parameter fit in the plane $\left(\Omega_{\mathrm{m}}, w_{0}\right)$ (see also Table 4$)$. The solid lines represent $68 \%(1 \sigma), 95 \%(2 \sigma)$, and $99 \%$ CL contours.

as: $\sigma(m)_{\text {tot }, i}^{2}=\sigma(m)_{\text {disp }}^{2} / N_{i}+\sigma(m)_{\text {irr }}^{2}$ where $N_{i}$ is the number of SNIa in the ith 0.1 redshift bin. In the case of SNAP, $\sigma(m)_{\text {irr }}$ is equal to 0.02 .

The SNAP mission also plans a large cosmic shear survey. The possibilities for measuring a constant equation of state parameter $w$ with lensing data were studied by Rhodes et al. (2004), Massey et al. (2004), and Réfrégier et al. (2004). We here extend the study to the case of an evolving equation of state. We use the same assumptions in the simulation as in Réfrégier et al. (2004) with a measurement of the lensing power spectrum in 2 redshift bins, except for the survey size, which has increased from $300^{\circ 2}$ to $1000^{\circ 2}$ (Aldering et al. 2004) and for the more conservative range of multipoles $\ell$ considered (see Sect. 4.1).

The long-term survey parameters are summarized in Table 3 .

\subsection{Results}

The combination of the three data sets is performed with, and without, a redshift variation for the equation of state, for both mid-term and long-term data sets.

The different plots in Fig. 3 show the results for individual mid-term probes and for their combination. The results are for a constant $w_{0}$, plotted as a function of the matter density $\Omega_{\mathrm{m}}$.

The SNLS survey combined with the nearby sample will improve the present precision on $w_{0}$ by a factor 2 . The expected contours from cosmic shear have the same behavior as the CMB but provide a slightly better constraint on $\Omega_{\mathrm{m}}$ and a different correlation with $w_{0}$; $\mathrm{CMB}$ and weak lensing data have a positive $\left(w_{0}, \Omega_{\mathrm{m}}\right)$ correlation compared to SNIa data, which have a negative correlation. This explains the impressive gain when the three data sets are combined, as shown in Table 4. Combining WMAP with Olimpo data helps to constrain $w_{0}$ through the correlation matrix as Olimpo expects to have more information for the large $\ell$ of the power spectrum.

Figure 4 gives the expected accuracy of the mid-term surveys on the parameters of an evolving equation of state. The CL contours plots of $w_{a}$ versus $w_{0}$ are obtained with a 9-cosmological parameter fit. Here also, we observe good complementarity: there is little information on the time evolution from SNIa with no prior, while the large redshift range from CMB data is adding a strong anti-correlated constraint on $w_{a}$.

A combined analysis proves far superior to analysis with only SNIa. In the favorable case, where we add more SNIa from the HST survey, we expect a gain of a factor 2 on the errors, but it is not enough to lift degeneracies, so the expected precision on $w_{a}$ with these data will not be sufficient to answer questions about the nature of the dark energy.

We have distinguished three scenarii (today/mid-term/longterm). We may have considered a hybrid configuration (expected in 2009-2010) with Planck + ground observations for SN and WL. Unfortunately, the gain with respect to the midterm scenario is small. Therefore, for the sake of simplicity, we 

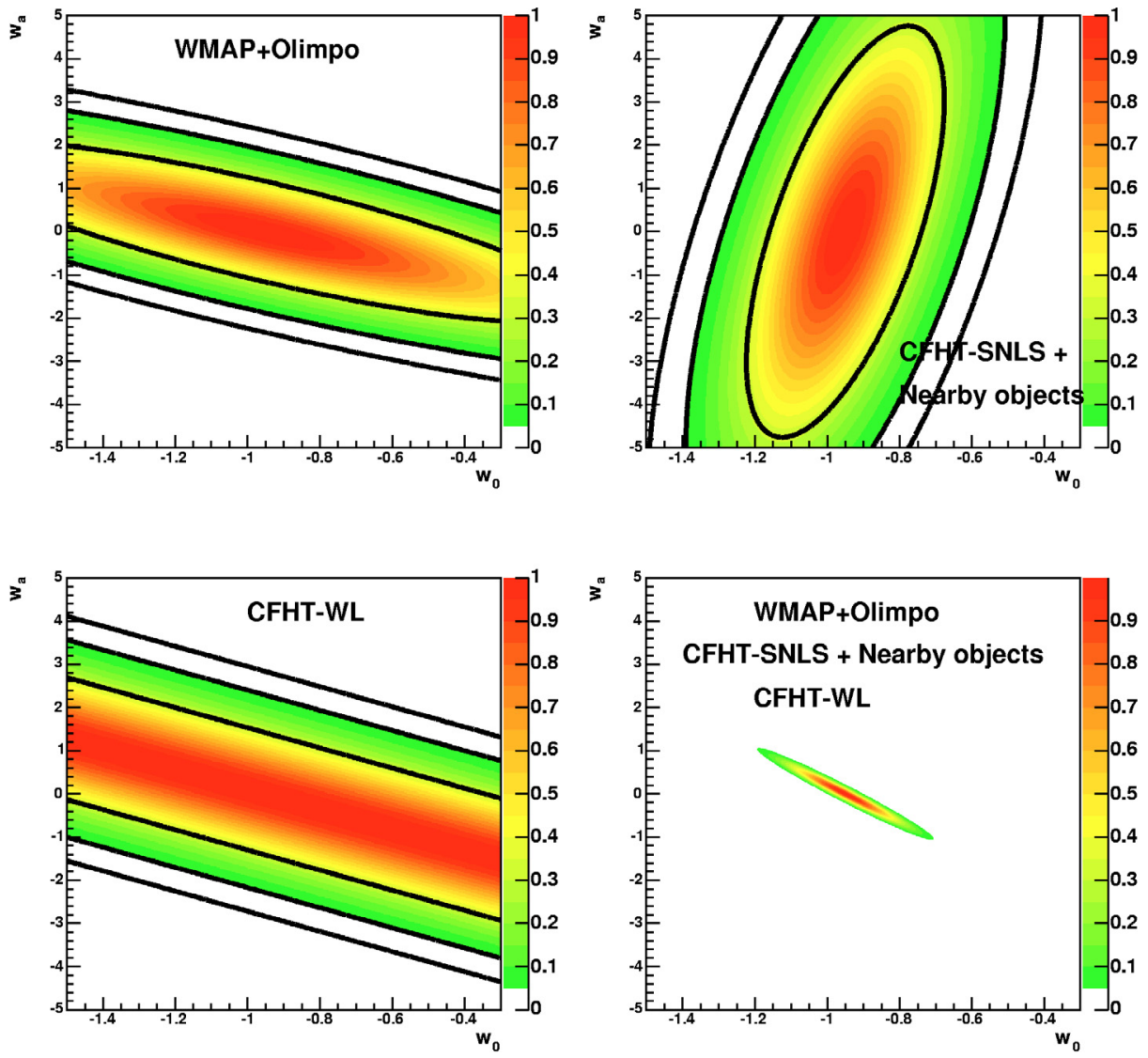

Fig. 4. The CL contours for mid-term CMB (WMAP+Olimpo), SNIa, and weak lensing data from CFHTLS and the combination of the three probes for the 9-parameter fit in the plane $\left(w_{0}, w_{a}\right)$ (see also Table 4). The solid lines represent $68 \%(1 \sigma), 95 \%(2 \sigma)$, and $99 \%$ CL contours.

Table 4. Expected sensitivity on cosmological parameters for three scenarii: Current supernova and CMB experiments (WMAP and Riess et al. 2004), mid-term experiments (CFHT-SNLS (supernova surveys), CFHTLS-WL (weak lensing) and CMB (WMAP+Olimpo)), long term experiments (CMB (Planck) and SNAP (supernovae and weak lensing)). For each scenario, the first column gives the $1 \sigma$ error computed with the Fisher matrix techniques for the 8 free parameter configuration and the second columns gives the $1 \sigma$ error for the 9 free parameter configuration. NB: the CL may vary with the fiducial model.

\begin{tabular}{l|cccccc}
\hline \hline Scenario & \multicolumn{3}{|c}{ Today } & \multicolumn{2}{c}{ Mid-term } & \multicolumn{2}{c}{ Long-term } \\
\hline$\Omega_{\mathrm{b}}$ & 0.003 & 0.004 & 0.001 & 0.002 & 0.0008 & 0.0008 \\
$\Omega_{\mathrm{m}}$ & 0.04 & 0.04 & 0.01 & 0.01 & 0.004 & 0.004 \\
$h$ & 0.03 & 0.03 & 0.01 & 0.01 & 0.006 & 0.006 \\
$n_{s}$ & 0.03 & 0.03 & 0.006 & 0.009 & 0.003 & 0.003 \\
$\tau$ & 0.05 & 0.04 & 0.01 & 0.01 & 0.01 & 0.01 \\
$w_{0}$ & 0.11 & 0.22 & 0.02 & 0.10 & 0.02 & 0.04 \\
$w_{a}$ & - & 0.99 & - & 0.43 & - & 0.07 \\
$A$ & 0.10 & 0.10 & 0.02 & 0.02 & 0.02 & 0.02 \\
$M_{s 0}$ & 0.03 & 0.03 & 0.01 & 0.01 & 0.01 & 0.01 \\
\hline
\end{tabular}

have only considered a long-term scenario with only satellite observations.

The inclusion of future space missions with multiple $z \mathrm{WL}$ lensing data, combined to future SNIa and CMB data, shows an improved sensitivity for all parameters, and in particular, for the time evolution of the equation of state. The expected
Table 5. Expected sensitivity on cosmological parameters for the long-term missions with CMB (Planck) and SNAP (supernova surveys and weak lensing) for the 9-free parameter configuration.

\begin{tabular}{l|ccc}
\hline \hline Model & $\Lambda$ CDM & SUGRA & Phantom \\
\hline$\Omega_{\mathrm{b}}$ & 0.0008 & 0.0008 & 0.0007 \\
$\Omega_{\mathrm{m}}$ & 0.004 & 0.004 & 0.003 \\
$h$ & 0.006 & 0.006 & 0.005 \\
$n_{S}$ & 0.003 & 0.003 & 0.003 \\
$\tau$ & 0.01 & 0.01 & 0.01 \\
$w_{0}$ & 0.04 & 0.04 & 0.03 \\
$w_{a}$ & 0.07 & 0.06 & 0.14 \\
$A$ & 0.02 & 0.02 & 0.02 \\
$M_{s 0}$ & 0.015 & 0.014 & 0.013 \\
\hline
\end{tabular}

accuracy on $w_{a}$ for the different combinations is summarized in Table 4. There is again large improvement from the combination of the three data sets. The precision for the long-term surveys will be sufficient to discriminate between the different models chosen, as shown in the top plot of Fig. 5 and in Table 5, while this is not the case for the mid-term surveys. This figure illustrates, moreover, that the errors on $w_{a}$ and $w_{0}$ and the correlation between these two variables are strongly dependent on the choice of the fiducial model.

More generally, the combination of the probes with the full correlation matrix allows the extraction of the entire information available. For instance, the large correlation between $n_{S}$ 

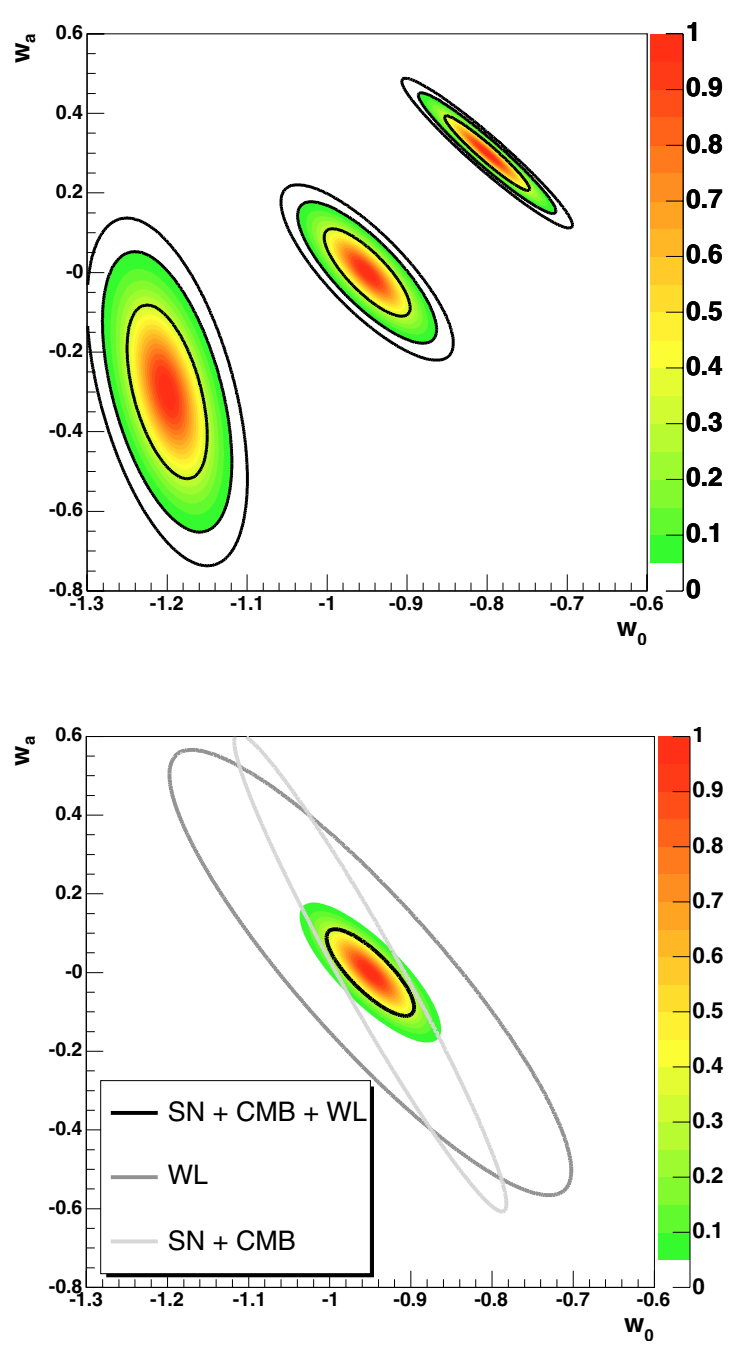

Fig. 5. The CL contours for future space data from SNAP (SNIa and $\mathrm{WL})$ and Planck $(\mathrm{CMB})$ for a 9-parameter fit in the plane $\left(w_{0}, w_{a}\right)$. The top plot shows the combination of SNAP (SNIa+WL) and CMB for three different models ( $\Lambda \mathrm{CDM}$, SUGRA, and Phantom). The solid lines represent $68 \%(1 \sigma), 95 \%(2 \sigma)$, and $99 \%$ CL contours. The bottom plot shows the CL for the combined three long-term probes. The solid lines are the $1 \sigma$ contours for different combinations: WL alone, combined SNIa and CMB, and the three combined probes.

and $w_{a}$ observed for the weak lensing probe combined with the precise measurement of $n_{s}$ given by the CMB, gives a better sensitivity on $w_{a}$ than the simple combination of the two $w_{a}$ values, obtained separately for the CMB and weak lensing. Such an effect occurs for several other pairs of cosmological parameters considered in this study. The bottom plot of Fig. 5 is an illustration of this effect. It shows the combination of the 3 probes in the $\left(w_{0}, w_{a}\right)$ plane. The $1 \sigma$ contour for the combined three probes is more constraining than the 2-D combination of the three probes in the $\left(w_{0}, w_{a}\right)$ plane.

Finally, in the long-term scenario, the weak lensing probe provides a sensitivity on the measurement of $\left(w_{0}, w_{a}\right)$ that is comparable to those of the combined SN and CMB probes, whereas in the mid-term scenario the information brought by weak lensing was marginal. This large improvement observed in the information provided by the weak lensing, can be explained by the larger survey size and the deeper volume probed by SNAP/JDEM, compared to the ground CFHTLS WL survey. We thus conclude that adding weak lensing information will be an efficient way to help distinguishing between dark energy models. If systematic effects are controlled well, the future dedicated space missions may achieve a sensitivity of order 0.1 on $w_{a}$.

The SNAP/JDEM space mission is designed, in principle, to control its observational systematic effects for SNIa to the percent level, which is probably impossible to achieve in future ground experiments. In this study, we assign an irreducible systematic error on SNIa magnitudes of 0.02 , and systematic effects have been neglected for CMB and weak lensing. This can have a serious impact on the final sensitivity, in particular on the relative importance of each probe.

Other probes, whose combined effects we have not presented in this paper but intend to deal with in forthcoming studies, will therefore be very useful. For example, the recent evidence of baryonic oscillations (Eisenstein et al. 2005) is proof that new probes can be found. The present constraints that these results provide do not improve the combined analysis we present here. However, getting similar results from different probes greatly contributes to the credibility of a result, in particular when the systematical effects can be quite different, as is the case for the different probes we consider. Finally, the joint analysis of cluster data observed simultaneously with WL, SZ effect, and X-rays will allow the reduction of the intrinsic systematics of the WL probe.

\section{Conclusions}

In this paper, we have presented a statistical method based on a frequentist approach combining different cosmological probes. We have taken into account the full correlations of parameters without any priors and without the use of Markov chains.

Using current SNIa and WMAP data, we first fitted a parameterization of an evolving equation of state and found results in good agreement with other studies in the literature, thus checking our program. We confirm that data prefer a value of $w$ less than -1 but still agree well with the $\Lambda$ CDM model. We emphasize the impact of the implementation of the dark energy perturbations. This can explain the discrepancies in the central values found by various authors. We performed a complete statistical treatment, evaluated the errors for existing data, and checked that the Fisher approximation is close to the global treatment, as long as the parameters $\left(w_{0}, w_{a}\right)$ are in the "physical" region imposed by the CMB boundary condition: $w(z \rightarrow \infty)<0$.

We then used the Fisher approximation to calculate the expected errors for current surveys on the ground (e.g., CFHTLS) combined with CMB data, and compared them with the expected improvements from future space experiments. We confirm that the inclusion of multiple $z$ weak lensing data in a complete combination of the three probes is very powerful for the extraction of a constant $w$. However, a second generation of experiments like the Planck and SNAP/JDEM space missions is required, to access the variation of the equation of state with redshift at the 0.1 precision level. This level of precision needs 
to be confirmed by further studies of systematical effects, especially for weak lensing. Finally, the precision will also be degraded, if dark energy perturbations are included, but this remains a theoretical issue.

Acknowledgements. The authors are most grateful to M. Doran for the CMBEASY package, the only code that was not developed by this collaboration, and for his readiness to answer all questions. They wish to thank A. Amara, J. Bergé, A. Bonissent, D. Fouchez, F. Henry-Couannier, S. Basa, J.-M. Deharveng, J.-P. Kneib, R. Malina, C. Marinoni, A. Mazure, J. Rich, and P. Taxil for their contributions to stimulating discussions.

\section{References}

Aldering, G., et al. (SNAP Collaboration) 2004 [arXiv: astro-ph/0405232]

Bacon, D. J., Réfrégier, A., \& Ellis, R. 2000, MNRAS, 318, 625

Bacon, D. J., Réfrégier, A., Clowe, D., \& Ellis, R. 2001, MNRAS, 325,1065

Bardeen, J. M., Bond, J. R., Kaiser, N., \& Szalay, A. S. 1986, ApJ, 304,15

Bartelmann, M., \& Schneider, P. 2001, Phys. Rep., 340, 291

Benabed, K., \& Van Waerbeke, L. 2004, Phys. Rev. D, 70, 123515

Bennett, C., Halpern, M., Hinshaw, G., et al. (WMAP Collaboration) 2003, ApJS, 148, 1

Brax, P., \& Martin, J. 1999, Phys. Lett. B, 468, 40

Bunn, E., \& White, S. 1997, ApJ, 480, 6

Bridle, S. L., Lahav, O., Ostriker, J. P., \& Steinhardt, P. J. 2003, Science, 299, 1532

Caldwell, R. R. 2002, Phys. Lett. B, 545, 23

CFHTLS: see, for example, http://cfht . hawai .edu/Science/ CFHTLS-OLD/history_2001.html and http://cfht.hawaii.edu

Caldwell, R. R., \& Doran, M. 2005 [arXiv:astro-ph/0501104]

Carlin, B. P., \& Louis, T. A. 1996, Bayes and Empirical Bayes Methods for Data Analysis (London: Chapman and Hall)

Carroll, S. M., et al. 2005, Phys. Rev. D, 71, 063513

Chevallier, M., \& Polarski, D. 2001, Int. J. Mod. Phys. D, 10, 213

Christensen, N., \& Meyer, R. 1998, Phys. Rev. D, 58, 082001

Cooray, A., \& Hu, W. 2001, ApJ, 554, 56

Corasaniti, P., Kunz, M., Parkinson, D., Copeland, E. J., \& Bassett, B. A. 2004, Phys. Rev. D, 70, 083006

Doran, M. 2003 [arXiv: astro-ph/0302138]

Douspis, M., Riazuelo, A., Zolnierowski, Y., \& Blanchard, A. 2003, A\&A, 405, 409

Eisenstein, D. J., Zehavi, I., Hogg, D. W., Scoccimarro, R., et al. 2005 [arXiv: astro-ph/0501171]

Fisher, R. A. 1935, J. Roy. Stat. Soc., 98, 39

Feldman, G. J., \& Cousins, R. D. 1998, Phys. Rev. D, 57, 3873

Gerke, B. F., \& Efstathiou, G. 2002, MNRAS, 335, 33

Gilks, W. R., Richardson, S., \& Spiegelhalter, D. J. 1996, Markov Chain Monte Carlo in Practice (London: Chapman and Hall)

Gong, Y. G., \& Zhang, Y.-Z. 2005, Phys. Rev. D, 72, 043518

Hawkins, E., et al. 2003, MNRAS, 347, 78

Heymans, C., Van Waerbeke, L., Bacon, D., et al. 2005 [arXiv: astro-ph/0506112]

Hinshaw, G., Spergel, D. N., Verde, L., et al. 2003, ApJS, 148, 135

Hoekstra, H., Yee, H., \& Gladders, M. 2002, New Astron. Rev., 46, 767

Hu, W. 1999, ApJ, 522, L21

Hu, W. 2002, Phys. Rev. D, 65, 023003

Hu, W. 2004 [arXiv:astro-ph/0407158]
Hu, W., \& Tegmark, M. 1999, ApJ, 514, L65

Huterer, D. 2001, Phys. Rev. D, 65, 063001

Huterer, D., \& Turner, M. S. 2001, Phys. Rev. D, 64, 123527

Ishak, M. 2005 [arXiv:astro-ph/0501594]

James, F. 1978, CERN Program Library Long Writeup, D506

Kaiser, N. 1998, ApJ, 498, 26

Kim, A. G., Linder, E. V., Miquel, R., Mostek, N., et al. 2004, MNRAS, 347, 909

Knop, R. A., Aldering, G., Amanullah, R., et al. 2003, ApJ, 598, 102

Knox, L. 1995, Phys. Rev. D, 52, 4307

Lewis, A., \& Bridle, S. 2002, Phys. Rev. D, 66, 103511

Linder, E. V. 2003, Phys. Rev. Lett., 90, 091301

Linder, E. V., \& Jenkins, A. 2003, MNRAS, 346, 573

Linder, E. V., \& Huterer, D. 2005, Phys. Rev. D, 72, 043509

Lue, A., Scoccimaro, R., \& Starkman, G. 2004, Phys. Rev. D, 69, 044005

Ma, C.-P., Caldwell, R. R., Bode, P., \& Wang, L. 1999, ApJ, 521, L1

Maor, I., Brustein, R., Steinhardt, P. J., et al. 2001, Phys. Rev. Lett., 86,6

Maor, I., Brustein, R., McMahon, J., \& Steinhardt, P. J. 2002, Phys. Rev. D, 65, 123003

Masi, S., Ade, P., de Bernardis, P., et al. (Olimpo Collaboration) 2003, Mem. S.A.It., 74, 96

Massey, R., Rhodes, J., Refregier, A., et al. 2004, AJ, 127, 3089

Melchiorri, A., Mersini, L., Ödman, C. J., \& Trodden, M. 2003, Phys. Rev. D, 68, 043509

Mellier, Y., et al. 2002, SPIE Conf., 4847, Astronomical Telescopes and Instrumentation, Kona, August 2002, preprint [arXiv: astro-ph/0210091]

Neyman, J. 1937, Phil. Trans. Royal Soc. London, Series A, 236, 333

Peacock, J. A., \& Dodds, S. J. 1996, MNRAS, 280, L19

Peacock, J. A. 1997, MNRAS, 284, 885

Peebles, P. J. E., \& Ratra, R. 1988, ApJ, 325, L17

Rapetti, D., Allen, S., \& Weller, J. 2004, MNRAS, accepted [arXiv:astro-ph/0409574]

Réfrégier, A. 2003, ARA\&A, 41, 645

Réfrégier, A., Massey, R., Rhodes, J., et al. 2004, AJ, 127, 3102

Rhodes, J., Refregier, A., Collins, N. R., et al. 2004, ApJ, 605, 29

Riess, A. G., Strolger, L.-G., Tonry, J., et al. 2004, ApJ, 607, 665

Seljak, U., \& Zaldarriaga, M. 1996, ApJ, 469, 437

Seljak, U., Makarov, A., McDonald, P., et al. [arXiv: astro-ph/0407372]

Spergel, D. N., Verde, L., Peiris, H. V., et al. 2003, ApJS, 148, 175

SNLS, cf. e.g., http://cfht.hawaii.edu_SNLS

Sugiyama, N. 1995, ApJS, 100, 281

Sunyaev, R. A., \& Zeldovich, Ya. B. 1980, ARA\&A, 18, 537

Tauber, J. A., et al. (Planck Collaboration) 2004, Adv. Space Res., 34, 491

Tilquin, A. 2003, http://marwww.in2p3.fr/renoir/Kosmoshow.html

Tegmark, M., Strauss, M. A., Blanton, M. R., et al. 2004, Phys. Rev. D, 69, 103501

Upadhye, A., Ishak, M., Steinhardt, P. J., et al. [arXiv: astro-ph/0411803]

Verde, L., Peiris, H. V., Spergel, D. N., et al. 2003, ApJS, 148, 195

Virey, J.-M., Taxil, P., Tilquin, A., et al. 2004a, Phys. Rev. D, 70, 043514

Virey, J.-M., Ealet, A., Tao, C., et al. 2004b, Phys. Rev. D, 70, 121301

Wang, Y., \& Tegmark, M. 2004, Phys. Rev. Lett., 92, 241302

Weller, J., \& Albrecht, A. 2002, Phys. Rev. D, 65, 103512

Weller, J., \& Lewis, A. M. 2003, MNRAS, 346, 987

Wetterich, C. 1988, Nucl. Phys. B, 302, 668

Wood-Vasey, W. M., et al. (Nearby Supernova Factory) 2004, New Astron. Rev., 48, 637

Xia, J.-Q., Feng, B., \& Zhang, X.-M. 2004

[arXiv: astro-ph/0411501]

Zech, G. 2002, Eur. Phys. J. direct C, 4, 12 